\title{
Entschließung vom 6. Juni 2018
}

Die Zeit der Verantwortungslosigkeit ist vorbei: EuGH bestätigt gemeinsame Verantwortung von Facebook und Fanpage-Betreibern

Die unabhängigen Datenschutzbehörden des Bundes und der Länder begrüßen das Urteil des Europäischen Gerichtshofs (EuGH) vom 5. Juni 2018, das ihre langjährige Rechtsauffassung bestätigt.

Das Urteil des EuGH zur gemeinsamen Verantwortung von Facebook und den Betreibern einer Fanpage hat unmittelbare Auswirkungen auf die Seitenbetreiber. Diese können nicht mehr allein auf die datenschutzrechtliche Verantwortung von Facebook verweisen, sondern sind selbst mitverantwortlich für die Einhaltung des Datenschutzes gegenüber den Nutzenden ihrer Fanpage.

Dabei müssen sie die Verpflichtungen aus den aktuell geltenden Regelungen der Datenschutz-Grundverordnung (DSGVO) beachten. Zwar nimmt das Urteil Bezug auf die frühere Richtlinie 95/46/EG zum Schutz natürlicher Personen bei der Verarbeitung personenbezogener Daten zum freien Datenverkehr, doch die vom EuGH festgestellte Mitverantwortung der Seitenbetreiber erstreckt sich auf das jeweils geltende Recht, insbesondere auf die in der DS-GVO festgeschriebenen Rechte der Betroffenen und Pflichten der Verarbeiter.

Im Einzelnen ist Folgendes zu beachten:

- Wer eine Fanpage besucht, muss transparent und in verständlicher Form darüber informiert werden, welche Daten zu welchen Zwecken durch Facebook und die Fanpage-Betreiber verarbeitet werden. Dies gilt sowohl für Personen, die bei Facebook registriert sind, als auch für nicht registrierte Besucherinnen und Besucher des Netzwerks.

- Betreiber von Fanpages sollten sich selbst versichern, dass Facebook ihnen die Informationen zur Verfügung stellt, die zur Erfüllung der genannten Informationspflichten benötigt werden.

- Soweit Facebook Besucherinnen und Besucher einer Fanpage durch Erhebung personenbezogener Daten trackt, sei es durch den Einsatz von Cookies oder vergleichbarer Techniken oder durch die Speicherung der IP-Adresse, ist grundsätzlich eine Einwilligung der Nutzenden erforderlich, die die Anforderung der DS-GVO erfüllt.

- Für die Bereiche der gemeinsamen Verantwortung von Facebook und FanpageBetreibern ist in einer Vereinbarung festzulegen, wer von ihnen welche Verpflichtung der DS-GVO erfüllt. Diese Vereinbarung muss in wesentlichen Punkten den Betroffenen zur Verfügung gestellt werden, damit diese ihre Betroffenenrechte wahrnehmen können.

Für die Durchsetzung der Datenschutzvorgaben bei einer Fanpage ist die Aufsichtsbehörde zuständig, die für das jeweilige Unternehmen oder die Behörde zuständig ist, die die Fanpage betreibt. Die Durchsetzung der Datenschutzvorgaben im Verantwortungsbereich von Facebook selbst obliegt primär der irischen Datenschutzaufsicht im Rahmen der europäischen Zusammenarbeit.

Die deutschen Aufsichtsbehörden weisen darauf hin, dass nach dem Urteil des EuGH dringender Handlungsbedarf für die Betreiber von Fanpages besteht. Dabei ist nicht zu verkennen, dass die Fanpage-Betreiber ihre datenschutzrechtlichen Verantwortung nur erfüllen können, wenn Facebook selbst an der Lösung mitwirkt und ein datenschutzkonformes Produkt anbietet, das die Rechte der Betroffenen wahrt und einen ordnungsgemäßen Betrieb in Europa ermöglicht.

\section{Über die Datenschutzkonferenz (DSK)}

Die Datenschutzkonferenz besteht aus den unabhängigen Datenschutzbehörden des Bundes und der Länder. Sie hat das Ziel, die Datenschutzgrundrechte zu wahren und zu schützen, eine einheitliche Anwendung des europäischen und nationalen Datenschutzrechts zu erreichen und gemeinsam für seine Fortentwicklung einzutreten. 